\title{
Hepatocarcinoma con invasión cardiovascular: comunicación de 5 casos y revisión de la literatura
}

\author{
José $M$ Valera $M^{1}$, Roberto Merino $Z^{1}$, Patricio Palavecino $R^{2}$, \\ Luis Sepúlveda $M^{3}$, Gladys Smok $S^{4}$, Manuel Fernández $A^{5}$, \\ Javier Brahm B ${ }^{1,6}$. \\ Hepatocellular carcinoma with \\ cardiovascular invasion. \\ Report of five cases
}

The diagnosis of hepatocellular carcinoma in cirrhotic patients has increased, mainly due to early detection using newer imaging techniques. The therapeutic approach depends on the tumor staging and the liver function. Cardiac involvement has a very bad prognosis. We report three males aged 59, 75 and 76 years and two females, aged 64 and 79 years. All had cirrhosis of diverse aetiologies with hepatocellular carcinoma and tumoral invasion of the inferior vena cava and right atrium. Three patients died during hospital stay and two were discharged for conservative management at home. This rare complication has to be considered in cirrhosis (Rev Méd Chile 2004; 132: 1517-22).

(Key Words: Carcinoma, hepatocellular; Venous thrombosis)

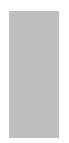
Recibido el 10 de junio, 2004. Aceptado el 31 de agosto, 2004.
${ }^{1}$ Sección de Gastroenterología y Departamentos de ${ }^{2}$ Radiología, ${ }^{3}$ Cardiología y ${ }^{4}$ Anatomía Patológica, Hospital Clínico Universidad de Chile y Departamentos de ${ }^{5}$ Radiología y ${ }^{6}$ Gastroenterología, Clínica Las Condes, Santiago.

$\mathrm{E}^{1}$ carcinoma hepatocelular $(\mathrm{CHC})$ representa más de $5 \%$ de todos los cánceres en el mundo y las muertes relacionadas con éste se estiman en 500 mil por año ${ }^{1}$. Este tumor puede debutar sintomáticamente con descompensación de la cirrosis de base, o ser de sospecha en pacientes asintomáticos por estudio de imágenes 0 medición de alfafetoproteína (AFP). En los países desarrollados se ha visto un aumento de su incidencia en el último tiempo, probablemente

Correspondencia a: Dr. Javier Brahm B. Sección Gastroenterología, Hospital Clínico Universidad de Chile. Avda. Santos Dumont 999, Santiago, Chile. Fono: 6788350. Fax: 6788349. E-mail: gastroen@ns.hospital.uchile.cl relacionado con la mejoría de exámenes diagnósticos y por la inmigración proveniente de zonas de alta prevalencia. Por otro lado, el mejor manejo de los pacientes cirróticos y la consiguiente mayor expectativa de vida de éstos, favorece su aparición, con una sobrevida que en casos avanzados suele ser inferior a 3 meses $^{2,4}$.

El diagnóstico de $\mathrm{CHC}$ en pacientes cirróticos se basa actualmente en los criterios establecidos en Barcelona $^{3}$, que consideran la presencia de al menos dos técnicas de imagen que muestren un nódulo de 2 o más centímetros con hipervascularización, o por una sola imagen asociada a AFP $>400$ $\mathrm{ng} / \mathrm{ml}$. Se han desarrollado varias clasificaciones para determinar el estadio tumoral y la función 
hepática residual y así definir la conducta terapéuti$\mathrm{ca}^{5}$, considerándose la invasión cardiovascular como un predictor de muy mal pronóstico ${ }^{6,7}$.

Por lo infrecuente del compromiso cardíaco del CHC y por las escasas comunicaciones al respecto, se presentan 5 casos de pacientes con estas características.

Caso 1. Mujer de 64 años, con antecedentes de hipertensión arterial, diabetes mellitus 2 y cirrosis diagnosticada 2 años antes, con estudio de anticuerpos antinucleares (+) 1/160, elevación de IgG y biopsia hepática compatible con el diagnóstico de hepatitis autoinmune en etapa cirrótica. Además, con marcada hipertensión portal, presentaba ascitis, várices esofágicas e hiperesplenismo. Manteniéndose en control periódico, se objetivó en exámenes de rutina elevación de AFP hasta 200 $\mathrm{ng} / \mathrm{ml}$ (VN: 0 - 15) y ecografía sólo compatible con daño hepático crónico. Sin embargo, la resonancia magnética (RM) mostró un $\mathrm{CHC}$ que infiltraba venas suprahepáticas (VSH), vena cava inferior (VCI) y que se extendía hasta aurícula derecha. Se hospitalizó en la sección de gastroenterología por un cuadro de shock refractario a volumen, encefalopatía grado IV, ictericia y ascitis a tensión. El estudio del líquido ascítico descartó peritonitis bacteriana espontánea (PBE) y hemoperitoneo. Dado su mal pronóstico e imposibilidad terapéutica se realizó manejo conservador, falleciendo a los tres días de su ingreso.

Caso 2. Hombre de 76 años, con antecedentes de hipertensión arterial, cardiopatía coronaria con doble by-pass, tres años antes, alcoholismo y tabaquismo crónico abandonado en esa ocasión, en tratamiento con enalapril, atenolol y aspirina. Inició, dos semanas previo a su ingreso al servicio de cardiología, un cuadro caracterizado por astenia, adinamia, anorexia, fatigabilidad y palidez, junto con una baja de peso de seis kilos en los dos últimos meses. Además, refería dos episodios de deposiciones melénicas, asociados a lipotimia transitoria, que no se comprobaron durante la hospitalización.

Se realizó endoscopia digestiva alta en dos ocasiones, que no demostró evidencias de sangrado ni hallazgos patológicos, excepto várices esofágicas incipientes. El hemograma no mostraba alteraciones.
La ecografía abdominal mostró una hernia incisional de laparatomía de Kocher derecha no complicada. Se realizó una ecografía cervical por palparse masa paratraqueal izquierda, que confirmó nódulo hipoecogénico y vascularizado, con aspecto de adenopatía, que midió 13 x $18 \mathrm{~mm}$ y ateromatosis carotídea bilateral. La biopsia de esta masa cervical mostró tejido fibroadiposo y muscular con proceso inflamatorio crónico activo granulomatoso no necrotizante, con focos supurados. El cintigrama óseo no mostró lesión ósea primaria ni secundaria.

La tomografía axial computada (TAC) de abdomen-pelvis y radiografía de tórax no mostraron hallazgos concluyentes. Sin embargo, la ecocardiografía transtorácica (ETT) y transesofágica (ETE) demostraron una masa de grandes dimensiones con carácter de tumor, que ocupaba la mayor parte de la cavidad auricular derecha, la que estaba muy dilatada, además akinesia anterosepto-apical y alteración de la motilidad segmentaria de ventrículo izquierdo, asociado a una disminución de la función sistólica global con presión normal de arteria pulmonar.

La RM de tórax y abdomen mostró cirrosis, atrofia del lóbulo hepático izquierdo y ausencia de VSH izquierda, asociado a una gran trombosis tumoral de ésta, la que se prolongaba a VCI y aurícula derecha, por lo que planteó el diagnóstico de CHC, encontrándose niveles de AFP de 52,2 $\mathrm{ng} / \mathrm{ml}$.

Dado su gran compromiso general y mal pronóstico, la familia solicitó el alta del paciente.

Caso 3. Hombre de 59 años, con antecedentes de cirrosis alcohólica diagnosticada 7 años antes y abstinente desde entonces. Se le realizó una biopsia hepática en marzo de 1998 compatible con cirrosis y esteatohepatitis.

En control regular, se mantenía con leve elevación de transaminasas y AFP en rango normal. En ecografía abdominal de octubre 2002, se observó hepatoesplenomegalia y un nódulo de aspecto tumoral en lóbulo hepático derecho de $5 \times 7 \mathrm{~cm}$, sin ascitis. La RM confirmó este hallazgo sin otros focos tumorales. En diciembre de 2002 se realizó cirugía con resección del tumor hepático, el que midió 8-9 cm de diámetro, con extensión retroperitoneal y diafragmática, practicándose hepatectomía parcial y resección de diafragma. La biopsia confir- 
mó el CHC, cirrosis y esteatohepatitis, con infiltración de diafragma y glándula suprarrenal.

En control posterior se mantenía en regulares condiciones, con un TAC abdominal, a los 6 meses sin evidencias de recidiva tumoral. Evolucionó enflaquecido y debilitado, hospitalizándose en septiembre 2003 por encefalopatía y PBE, la que respondió a tratamiento habitual. La RM mostró extensa recidiva tumoral con compromiso de VSH, VCI y aurícula derecha (Figura 1), la que se complementó con ETT y ETE que confirmaron este hallazgo (Figura 2).

Dado su mal pronóstico se da de alta con manejo médico conservador, falleciendo de muerte súbita en su domicilio 15 días más tarde.

Caso 4. Paciente de 75 años de sexo masculino, con antecedentes de cirrosis alcohólica, que ingresó al servicio de urgencia por una descompensación con encefalopatía portal grado 3 y que durante la hospitalización presentó hemorragia por várices esofágicas, la que se controló con ligadura variceal. Evolucionó con síndrome hepatorrenal y falleció a los pocos días de su ingreso.

Se realizó una autopsia, previa autorización de la familia, que demostró cirrosis micro-macronodular y CHC multicéntrico con trombosis tumoral de la vena porta intra y extra hepática, vena esplénica, ilíacas primitivas, renales, VCI y superior, y de la aurícula derecha (Figuras 3a, b).

Caso 5. Mujer de 79 años, con antecedente de cardiopatía hipertensiva, insuficiencia cardíaca $\mathrm{CF}$ II-III y fibrilación auricular en tratamiento anticoagulante desde 5 años antes, junto con enalapril, digoxina y diuréticos. Ingresó a la unidad de intermedio por episodios de hemorragia digestiva baja en los últimos 10 días, con hemodinamia estable y sangre fresca al tacto rectal. Con hematocrito $24 \%$, se transfunden glóbulos rojos y se realizó endoscopia alta que mostró várices esofágicas de tercio distal grado II/IV sin estigmas de riesgo. En exámenes de laboratorio destacaba albúmina 2,6 $\mathrm{gr} / \mathrm{dl}$, colesterol total $128 \mathrm{mg} / \mathrm{dl}$, transaminasa glutámico oxalacética 2x y fosfatasas alcalinas 135 (valor máx $104 \mathrm{mg} / \mathrm{dl}$ ). Se suspendió la anticoagulación y se estudió con TAC de abdomen que mostró ascitis importante, hígado cirrótico con múltiples imágenes de sustitución de hasta $4 \mathrm{~cm}$ de diámetro sugerentes de $\mathrm{CHC}$, y trombosis tumoral de la VSH media, VCI y de la aurícula derecha. Se estudiaron marcadores virales para virus B y C siendo negativos y AFP en rango normal. Se decidió manejo conservador, falleciendo la paciente a la semana de su ingreso al hospital.

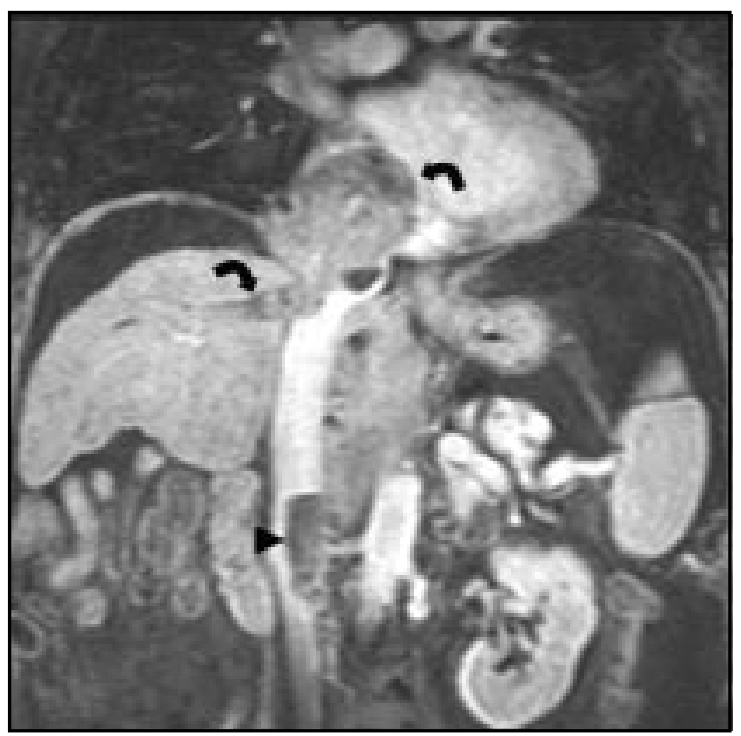

FIgURA 1. Corte coronal postinyección de contraste (gadolinio), que muestra la masa tumoral que se extiende desde la vena suprahepática a la aurícula, señalándose la invasión por el hepatocarcinoma de la vena cava inferior (cabeza de flecha) y de la aurícula derecha (flechas curvas).

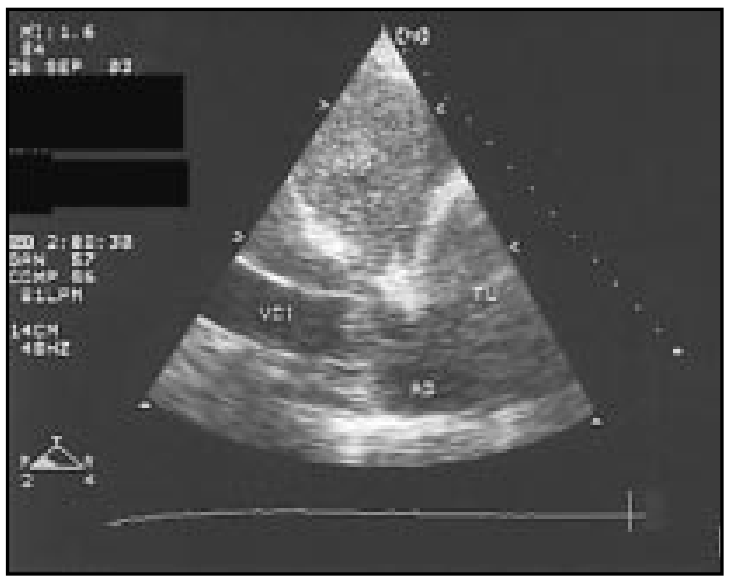

FiguRA 2. Ecocardiograma Doppler que muestra el tumor en aurícula derecha proveniente de vena cava superior y vena suprahepática, con leve dilatación de la aurícula derecha y resto de las cavidades normales. 


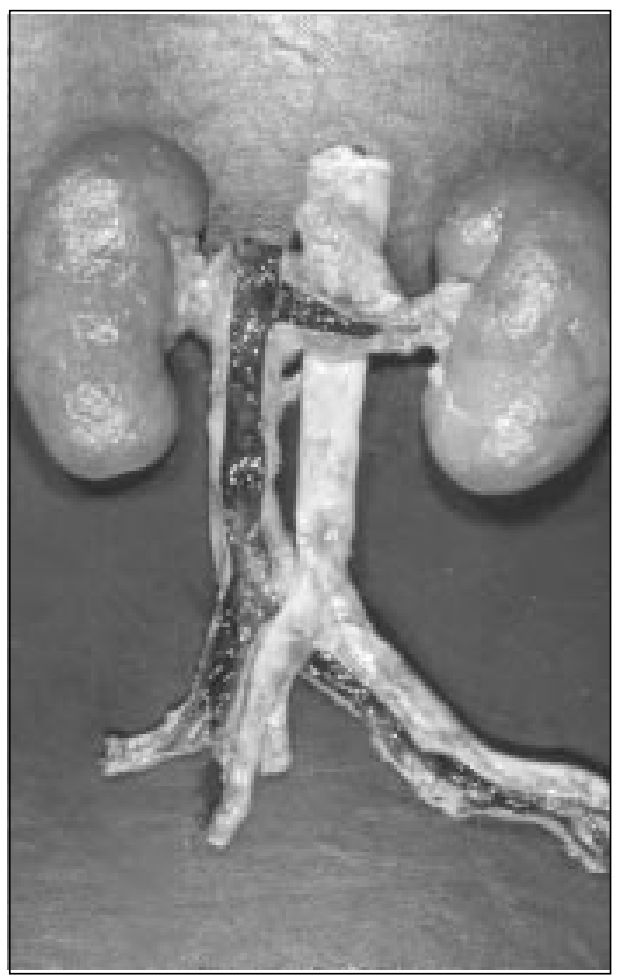

FiguRA 3b. Trombosis tumoral intracardíaca. Trombo tumoral adherido a endocardio (Hematoxilina-Eosina x 40).

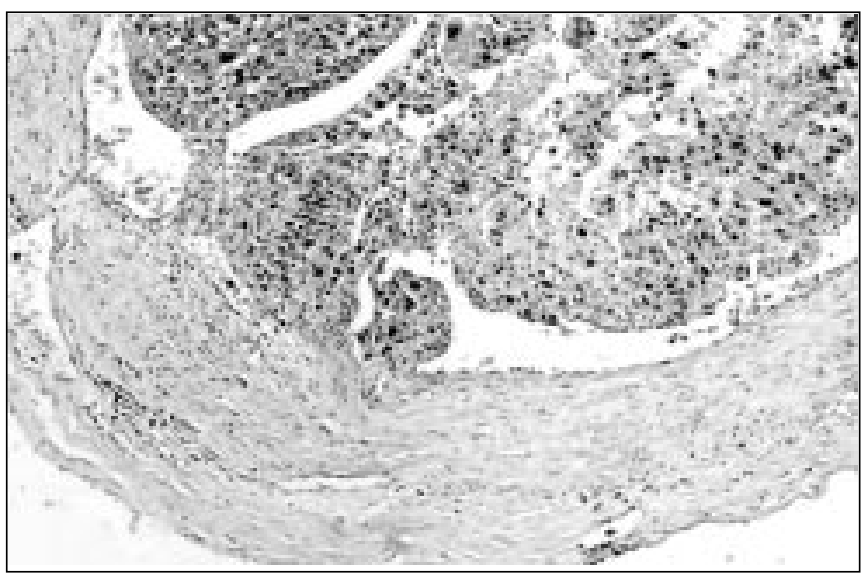

\section{DisCUSIÓN}

Los cinco casos clínicos expuestos representan distintas manifestaciones de $\mathrm{CHC}$. El primero en una paciente cirrótica conocida en seguimiento preventivo con ecografía abdominal y medición de alfafetoproteína cada 6 meses, el segundo como hallazgo cardiológico en un paciente en que se desconocía su enfermedad hepática de base, el tercero en un paciente cirrótico operado de $\mathrm{CHC}$ diez meses antes y que presenta PBE, el cuarto como hallazgo en el estudio necrópsico de un paciente fallecido por descompensación de su cirrosis con encefalopatía e insuficiencia renal, y el quinto en una paciente cardiópata que ingresa por una hemorragia digestiva alta de origen variceal (Tabla 1$)$.

El CHC en estadios avanzados tiene una probabilidad de invasión vascular de hasta $70 \%$ de los casos según estudios con necropsia y de $40 \%$ al momento del diagnóstico ${ }^{4}$. Ésta puede afectar la vena porta, la arteria y vena hepática, VCI, el árbol biliar e incluso la aurícula derecha ${ }^{10}$. La presencia de esta invasión vascular ha sido comunicada con mayor frecuencia en áreas de alta prevalencia como Japón ${ }^{10-12,17}$, siendo su presentación mucho más esporádica en occidente.

El compromiso cardiovascular afectando venas hepáticas, cava inferior y cavidades cardíacas condiciona un mal pronóstico y que puede tener fatales consecuencias. Esto debido al compromiso circulatorio y pulmonar por oclusión vascular ${ }^{8,9}$, obstrucción intracavitaria al flujo sanguíneo, disfunción valvular ${ }^{14}$ y embolias tanto pulmonares como sistémicas, lo que limita además las posibilidades terapéuticas que no han sido bien establecidas.

Se ha descrito la resección completa del $\mathrm{CHC}$ incluyendo la invasión de aurícula derecha, pero con una sobrevida de sólo 2 años complicada por recidiva tumoral ${ }^{12}$. La realización de cirugía de rescate, utilizando circulación extracorpórea por el alto riesgo de oclusión del tracto de salida del ventrículo derecho ${ }^{13}$, ocasionalmente ha tenido un resultado transitorio favorable ${ }^{14}$, pero no pare- 
Tabla 1. Características clínicas, exámenes, manejo y evolución de 5 casos de carcinoma hepatocelular con invasión cardiovascular

\begin{tabular}{|cclll|}
\hline Caso & $\begin{array}{c}\text { Edad/Sexo } \\
\text { (M=mujer } \\
\text { H=hombre })\end{array}$ & $\begin{array}{l}\text { Etiología } \\
\text { cirrosis }\end{array}$ & $\begin{array}{l}\text { Estudio } \\
\text { diagnóstico de } \\
\text { CHC metastásico }\end{array}$ & Manejo/Evolución \\
\hline 1 & $64 / \mathrm{M}$ & $\begin{array}{l}\text { Hepatitis } \\
\text { autoinmune }\end{array}$ & RM, AFP & Conservador/fallece \\
2 & $76 / \mathrm{H}$ & $\begin{array}{l}\text { OH } \\
\text { OH }\end{array}$ & $\begin{array}{l}\text { RM, ETT y ETE } \\
\text { RM, ETT y ETE }\end{array}$ & $\begin{array}{l}\text { Conservador, en control } \\
\text { Cirugía de CHC y recidiva al } \\
10 \text { o mes/fallece }\end{array}$ \\
3 & $59 / \mathrm{H}$ & Fallecido \\
4 & $75 / \mathrm{H}$ & OH & Necropsia & Conservador/fallece \\
5 & $79 / \mathrm{M}$ & Criptogénica & TAC & C.
\end{tabular}

OH: alcohólica; RM: resonancia magnética; AFP: alfafetoproteína; ETT y ETE: ecocardiograma transtorácico y transesofágico: TAC: tomografía computada de abdomen.

ce modificar el mal pronóstico a corto plazo ${ }^{11,18}$. En pacientes cirróticos, la insuficiencia hepática, coagulopatía y complicaciones postoperatorias dificultan más aún el manejo.

La ocupación tumoral de las cavidades cardíacas derechas por extensión del CHC puede estar subdiagnosticado, dada su escasa expresión clínica, siendo la ecocardiografía un examen útil en su diagnóstico y la vía transesofágica de mayor precisión para diferenciar el origen de la lesión ${ }^{15,16}$, como se observó en nuestros casos 2 y 3. Este examen puede, incluso, definir rápidamente el diagnóstico y la extensión intracavitaria en condiciones de urgencia, como en un caso comunicado con obstrucción de salida del ventrículo derecho durante la cirugía de un $\mathrm{CHC}$ con metástasis inicialmente auricular ${ }^{13}$. En algunos informes de casos similares, el diagnóstico se hizo en base a la $\mathrm{TAC}^{17}$, como en nuestra quinta paciente, destacan-

\section{REFERENCIAS}

1. Bosch FX, Ribes J, Borras J. Epidemiology of primary liver cancer. Semin Liver Dis 1999; 19: 271-85.

2. Deuffic S, Buffat L, Poynard $T$, Valeron AJ. Modeling the hepatitis $C$ virus epidemic in France. Hepatology 1999; 29: 1596-601. do en otros tres de nuestros casos la gran precisión diagnóstica obtenida con las imágenes de la RM, la que además evita el uso de contraste que puede afectar la función renal de estos pacientes. Más aún, en nuestros casos 1 y 2 , la TAC realizada contemporáneamente no evidenció el compromiso vascular tumoral.

Los casos expuestos anteriormente plantean la dificultad para realizar un diagnóstico correcto de invasión cardiovascular en casos de $\mathrm{CHC}$ y las eventuales limitaciones o ventajas que presentan algunas técnicas diagnósticas. Si bien fueron atendidos en centros de referencia, es notable la acumulación de estos casos, lo que podría deberse a un aumento en la detección de esta patología. Por lo anterior, la extensión vascular de un $\mathrm{CHC}$ debe considerarse en nuestro medio, dados los avances en imagenología y terapéutica para optimizar el manejo de estos pacientes. 
5. Befeier S, Di Bisceglie AM. Hepatocellular carcinoma: diagnosis and treatment. Gastroenterology 2002; 122: 1609-19.

6. Izum $R$, Shimizu K, Li T, Yagi M, Matsui O, Nonomura A Et AL. Prognostic factors of hepatocellular carcinoma in patients undergoing hepatic resection. Gastroenterology 1994; 106: 720-7.

7. Llovet JM, Bruix J, Fuster J, Castels A, GarcíaVALDECASAS JC, GRANDE L ET AL. Liver transplantation for treatment of small hepatocellular carcinoma: the TNM classification does not have prognostic power. Hepatology 1998; 27: 1572-7.

8. Chevret S, Trinchet JC, Mathieu D, Rached AA, Beaugrand M, Chastang C. A new prognostic classification for predicting survival in patients with hepatocellular carcinoma. J Hepatol 1999; 31: 133-41.

9. Llovet JM, Bustamante J, Castells A, Vilana R, Ayuso MC, SAla M ET al. Natural history of untreated nonsurgical hepatocellular carcinoma: rationale for the design and evaluation of therapeutic trials. Hepatology 1999; 29: 62-7.

10. Үoко $\mathrm{Y}$, HachiYa T, KuRachi K, Окамото K, TSUChiYa Y, Окumara T et al. Resection of Hepatocellular Carcinoma with A Tumor Thrombus Extending into the Right Atrium. Department of Surgery II, Hamamatsu University School of Medicine. Jpn J Gastroenterol Surg 2000; 33: 1507-11.

11. Goto H, Kaneko Y, Utoh J, Nishimura K, Miyauchi Y, Iwanaga K. Surgery of hepatoma with intracavitary cardiac extension. Heart Vessels 1986; 2: 60-2.
12. Fujisaki M, KuRihara E, KikUChi K, NishiKawa K, UEMATSU Y. Hepatocellular carcinoma with tumor thrombus extending into the right atrium: report of a successful resection with the use of cardiopulmonary bypass. Surgery 1991; 109: 214-9.

13. Peng Sy, Wu CC, Chang CP, Peng SK, Ho WM. Metastatic hepatocellular carcinoma of the right atrium causing right ventricular outflow tract obstruction during induction of anesthesia, a case report. Acta Anaesthesiol Sin 2002; 40: 205-8.

14. Chu MW, Aboguddah A, Kraus PA, Dewar LR. Urgent heart surgery for an atrial mass: metastatic hepatocellular carcinoma. Ann Thorac Surg 2001; 72: 931-3.

15. Chia BL, Choo MH, Tan L, Tan A, Oon CJ, Chew $\mathrm{PH}$. Two-dimensional echocardiographic abnormalities of right atrial metastatic tumors in hepatoma. Chest 1985; 87: 399-401.

16. Lynch M, Ciements SD, Shanewise JS, Chen CC, MARTIN RP. Right-sided cardiac tumors detected by transesophageal echocardiography and its usefulness in differentiating the benign from the malignant ones. Am J Cardiol 1997; 79: 781-4.

17. Takayasu $\mathrm{K}$, Muramatsu $\mathrm{Y}$, Furukawa $\mathrm{H}$. Intersegmental attenuation difference on CT caused by partial Budd-Chiari syndrome secondary to hepatocellular carcinoma: report of two cases. Department of Diagnostic Radiology, National Cancer Center Hospital, Tokyo, Japan. Jpn J Clin Oncol 1997; 27: 185-8.

18. Ehrlich DA, Widmann JJ, Berger RL, Abelmann WD. Intracavitary cardiac extension of hepatoma. Ann Thorac Surg 1975; 19: 206-11. 